\title{
Rhétorique de l'engagement ludique dans des dispositifs de ludification
}

\section{Maude Bonenfant et Thibault Philippette}

\section{(2) OpenEdition \\ 1 Journals}

Édition électronique

URL : http://journals.openedition.org/sdj/1422

DOI : $10.4000 /$ sdj. 1422

ISSN : 2269-2657

\section{Éditeur}

Laboratoire EXPERICE - Centre de Recherche Interuniversitaire Expérience Ressources Culturelles Education

\section{Référence électronique}

Maude Bonenfant et Thibault Philippette, «Rhétorique de l'engagement ludique dans des dispositifs de ludification », Sciences du jeu [En ligne], 10 | 2018, mis en ligne le 30 octobre 2018, consulté le 19 avril 2019. URL : http://journals.openedition.org/sdj/1422 ; DOI : 10.4000/sdj.1422

\section{Ce document a été généré automatiquement le 19 avril 2019}

Tous droits réservés 


\title{
Rhétorique de l'engagement ludique dans des dispositifs de ludification
}

\author{
Maude Bonenfant et Thibault Philippette
}

1 La notion d'engagement réfère à une disposition subjective qui motive la réalisation d'une action civique et, plus largement, de communication (Berger et De Munck, 2015 ; Bucy et Gregson, 2001). Dans cet ordre d'idées, s'engager dans un jeu consiste à poser le choix de participer à une expérience souhaitée. Or, la tendance actuelle de la ludification (Deterding et al., 2011) ou de la ludicisation (Genvo, 2013) tend à vouloir exploiter des ressorts ludiques afin de motiver les individus à s'engager de manière extrinsèque (Ryan et Deci, 2000) dans une activité a priori non ludique, par exemple, de consommation (Philippette, Collard, et Klein, 2016).

2 Traiter de la thématique de la ludification est une manière de questionner le rapport au « jeu» (Henriot, 1969, 1989) et à l'engagement dans celui-ci. À ce titre, comme nous le verrons, il faut distinguer l'engagement d'autres concepts comme celui d'immersion, de présence ou d'incorporation, en ce qu'il invite à la fois à la participation, mais également à la distance critique et à l'effort interprétatif (Douglas et Hargadon, 2000). Le but de cet article sera de problématiser la manière dont la ludification permet de conditionner un participant à entreprendre et poursuivre une activité dont les objectifs sont étrangers au jeu.

3 En nous questionnant sur la façon dont ces stratégies de ludification peuvent susciter l'engagement, nous nous demanderons tout d'abord, sur un plan théorique, comment lier ce concept de ludification avec la notion d'engagement. Ensuite, sur un plan plus pratique, nous nous interrogerons sur les éléments sémiotiques et discursifs participant d'une forme de «rhétorique» de ces dispositifs ludifiés. Nous proposerons une distinction entre rhétorique procédurale et rhétorique processuelle qui offre des visions complémentaires, mais différentes du jeu, vu comme un moyen permettant d'atteindre un certain état d'engagement, voire d'adhésion. Pour illustrer notre propos, nous proposerons quelques analyses de cas représentatifs des formes d'engagement décrites. Cette étude exploratoire ne prétend évidemment pas à l'exhaustivité, mais permettra de clarifier notre travail de conceptualisation. 
Pour conclure, nous interrogerons de manière plus critique l'éthique de la rhétorique ludique, qu'elle soit procédurale ou processuelle. Cette entreprise de déconstruction ne visera pas à condamner ou encenser le phénomène, mais à offrir quelques réflexions permettant de mieux problématiser ces stratégies ainsi que leurs effets si une forme d'engagement ou d'adhésion est effective.

\section{Gamification, ludification ou ludicisation?}

5 Le concept de "gamification", qui connaît un franc succès depuis plusieurs années, renvoie en principe à l'utilisation d'objets ludiques et de techniques de game design dans des contextes non ludiques (Werbach et Hunter, 2012). Ce concept a été largement défendu depuis la fin des années 2000 par des "gourous » en marketing en tant que moyen permettant de dégager du profit sur des marchés de plus en plus concurrentiels (Fuchs et al., 2014, Introduction). Pour Olivier Mauco (2012), prise d'un point de vue commercial, la gamification s'apparente en effet à un ensemble de techniques béhavioristes dans une économie de l'attention. Cependant, on peut considérer l'engouement pour ce phénomène comme relevant plus globalement d'un mouvement culturel, d'une forme de «tournant ludique » dans nos sociétés postmodernes (Raessens, 2014).

6 Le concept de "ludification ", parfois considéré comme traduction française synonyme de gamification - mais qui englobe en anglais d'autres significations (Bonenfant et Genvo, 2014) -, doit être plus largement considéré comme une tendance socioculturelle touchant à la fois l'extension pervasive de certains jeux, les différentes formes d'interactivités ludiques et l'utilisation de jeux dans des contextes préalablement «non ludiques ». Ce dernier aspect traitant à son tour aussi bien de contextes non ludiques ramenés dans des environnements de jeu (serious games) que de l'utilisation d'éléments de jeux - technologies, pratiques ou design - importés dans des contextes non ludiques. La gamification ne recouvre alors stricto sensu que ce dernier aspect de la conception et du design ludique de situations non ludiques (Deterding et al., 2011).

7 Malgré tout, la ludification est souvent assimilée à la gamification et viserait à utiliser l'état d'esprit ainsi que la mécanique du jeu pour résoudre des problèmes et faire participer les utilisateurs (Zichermann et Cunningham, 2011). Cette définition de la ludification ne doit pas être confondue avec la «ludicisation", qui renvoie plutôt au processus visant à faire entrer un objet dans la «sphère du jeu " (Genvo, 2012). Cette notion plus expérientielle, aux contours évolutifs, a le mérite de ne pas simplement limiter le jeu à un ensemble de techniques et de poser, de manière problématique et non évidente, la transformation d'un objet ou d'une situation « autre » en un jeu (Silva, 2013). Or, vouloir définir la ludicisation en tant qu'expérience, c'est - comme le notait Gilles Brougère à propos du jeu lui-même - une entreprise complexe dans laquelle il faut accepter de traiter avec des frontières floues (Brougère, 2005, p. 41).

Dans le cadre de cet article, nous parlerons de ludification en référence à l'utilisation de stratégies reconnues comme appartenant au domaine du jeu et qui sont utilisées dans des contextes extérieurs au jeu. À travers nos illustrations, nous tâcherons de varier les stratégies employées et les contextes auxquels elles s'appliquent. Le commun dénominateur étant de nous intéresser à des dispositifs ${ }^{1}$ qui utilisent des mécaniques de jeu et qui véhiculent en même temps des valeurs à propos de cette activité ludique. Il ne 
s'agit pas d'un travail empirique d'analyse des effets mesurables de ces dispositifs ou encore d'une typologie exhaustive, mais bien d'une réflexion en amont à propos de stratégies rhétoriques (cf. infra) basées sur la ludification et de leurs effets probables sur l'engagement des joueurs-participants.

\section{L'engagement tel qu'on en parle}

9 Étymologiquement, la notion d'engagement a un sens contractuel, voire juridique. Il s'agit de l'« action de mettre (qqch) en gage », « de lier (qqn), de se lier par une promesse ou une convention » ou de l'" état où l'on est engagé » (Le Grand Robert de la langue française, 2004). Qu'il y ait dépôt, promesse, serment, contrat, liaison ou mariage, l'engagement sous-entend une forme d'investissement matériel et affectif. Dans le domaine du marketing par exemple, l'engagement est une notion commune qui réfère à la loyauté du consommateur (investissement affectif) envers une marque ou un produit pour s'assurer de l'acte d'achat (investissement matériel).

Plusieurs entreprises font ouvertement la promotion de la ludification comme stratégie permettant de favoriser l'engagement du client ou de l'employé. Badgeville ${ }^{2}$ se présente comme "le leader en ludification d'entreprise" avec the engagement API. Hoopla ${ }^{3}$ est présenté comme une application qui vise à «motiver et engager son équipe grâce à la ludification». Dopamine, l'agence "créative» lancée par Gabe Zichermann ${ }^{4}$, affirme: "nous fabriquons des expériences ludifiées autour de l'ADN de votre marque ${ }^{5}$ ». L'argumentaire promu est celui d'un conditionnement des comportements afin de s'assurer un investissement affectif et matériel de la part du consommateur ou de l'employé.

11 De leur côté, au lieu de parler d'« engagement », les entreprises de jeux vidéo utilisent davantage le terme d'«immersion» comme argument publicitaire pour promouvoir un type d'expérience ludique qui serait à viser (Chabot, 2012). Néanmoins, il existerait une confusion sur la définition même du terme et de ce qui est entendu par «immersif». Citant le travail de Calleja (2011) concernant la polysémie du terme, Chabot relève plusieurs exemples issus de l'industrie vidéoludique où l'immersion dans un jeu est vantée tour à tour en référence au réalisme des images, à l'écran en trois dimensions, à des périphériques plus adaptés, voire à l'absence de périphériques que l'on manipule (e.g. Kinect sur la console Xbox), à une qualité propre aux « grands jeux » (notion éminemment floue, pleine de sous-entendus), etc.

12 Il faut dire que, même dans le champ des études du jeu, les notions d'engagement et d'immersion posent problème et aucune définition ne fait consensus. Généralement, ces deux notions sont abordées en parallèle (Ermi et Mäyrä, 2005; Poels, de Kort, et Ijsselsteijn, 2007), mais elles sont également distinguées de la notion de présence (Skalski, Lange, et Tamborini, 2006) pour faire état de l'expérience des joueurs (McMahan, 2003 ; Slater et al., 1996). De son côté, Huizinga parlait dès 1938 d'« absorption " pour qualifier la particularité de cet état psychique du joueur qui entre dans le « cercle magique » du jeu, ce qui ajoute un nouveau terme à la compréhension possible de l'engagement. 


\section{L'engagement comme concept psychologique}

13 Du côté de la psychologie, la notion d'engagement est associée positivement à une forme de bien-être (Brault-Labbé et Dubé, 2012), mais les théories psychologiques ont traité de différents types d'engagement (comportemental, affectif, moral, structurel, identitaire, etc.), souvent de façon isolée, dans différents domaines ou sphères de la vie sociale et humaine (Brault-Labbé et Dubé, 2012, p. 118) - ce qui rend cette notion extrêmement complexe à cerner du point de vue psychologique.

Une théorie psychologique particulière de l'engagement s'est imposée dans la sphère des études du jeu et a été largement reprise (Cowley et al., 2008; Shernoff et al., 2003) : la théorie de l'expérience optimale ou flow (Csikszentmihalyi, 2008). L'expérience de flow caractérise une variété de situations qui, indépendamment de questions de genre, de lieux ou même de culture, seraient rapportées de manière similaire dans des termes positifs. Pour Csikszentmihalyi, cet état de bonheur psychologique ne dépend pas tellement de facteurs extérieurs, mais bien de la façon dont on vit ces situations, qu'il appelle «ordre de conscience » et qui advient «[...] lorsque l'énergie psychique - ou attention - est investie dans des objectifs réalistes et lorsque les capacités correspondent aux opportunités d'action» (Csikszentmihalyi, 2008, p. 6, notre traduction). L'attention, qui permet la sélection des informations signifiantes, l'évaluation de la situation et les choix d'action, conduit à cette expérience optimale dès lors qu'elle est en harmonie avec les objectifs fixés. Alors, l'énergie psychique coule (flow) sans aucun effort (Csikszentmihalyi, 2008, p. 39). Cette théorie considère que le fait d'atteindre cette expérience de flow est caractéristique des individus autotéliques, c'est-à-dire qui sont naturellement et intrinsèquement motivés à faire ce qu'ils font (Csikszentmihalyi, 1997, p.117). Transposée à l'univers $\mathrm{du}$ jeu, cette théorie semble toutefois référer essentiellement à la dimension de "résolution de problème» de l'expérience ludique (Koster, 2005 ; McGonigal, 2011), ce qui n'en est qu'une composante, qui plus est variable d'un type de jeu à un autre.

Lorsque l'on se tourne vers les publications traitant de gamification, il est fait également référence à cette théorie psychologique du flow (Burke, 2014 ; Chatfield, 2010 ; Werbach et Hunter, 2012) comme forme d'état d'engagement optimal dans une activité. Pour essayer d'atteindre cet « état», les auteurs mobilisent d'autres concepts, comme celui de «boucle d'engagement " - le triptyque motivation, action, rétroaction - (Zichermann et Linder, 2013) ou la théorie de l'autodétermination (Werbach et Hunter, 2012). Cette dernière théorie (self-determination theory) de Deci et Ryan (2000) explique qu'entre une motivation forte et intrinsèque à faire quelque chose et l'absence complète de motivation, il existe une sorte de continuum de facteurs extrinsèques pouvant amener un individu à s'engager dans une activité. De manière succincte, le premier niveau, qu'ils appellent régulation externe, consiste à se soumettre aux ordres d'une autorité ou à vouloir bénéficier d'une récompense promise (e.g. recevoir des points et avantages). Le second, qu'ils appellent régulation introjectée, est une forme de contrôle de soi conditionné par l'idée que l'on se fait des coûts/bénéfices d'un comportement particulier (i.e. acceptation ou rejet social). Le troisième niveau, appelé régulation identifiée, résulte d'une prise de conscience de l'importance de certains actes ou de certaines activités (e.g. trier ses déchets). Enfin, le quatrième niveau, à la lisière de la motivation intrinsèque, s'appelle la régulation intégrée 
et caractérise une congruence entre l'image de soi et les comportements adoptés (e.g. écologiques).

\section{Le continuum de l'engagement}

Cette manière de considérer le continuum des types de motivation est parente avec les types d'engagement défendus dans des travaux précédents sur l'engagement et le jeu. Une de ces recherches, réalisées par théorisation ancrée (grounded theory), pose bien les bases de la réflexion. Leurs auteurs, Brown et Cairns (2004), présentent l'expérience ludique à partir de différents niveaux, l'engagement (involvement ${ }^{6}$ ) étant le premier stade avant l'absorption (engrossment) pour finalement atteindre l'immersion totale (total immersion). Leurs observations sont claires: pour qu'il y ait engagement, il faut un investissement de temps, d'effort et d'attention de la part du joueur ainsi qu'une manipulation appropriée, c'est-à-dire une combinaison entre l'intérêt du joueur de poursuivre l'activité ainsi que des contrôles et réponses du système adaptés à ce qui est exigé - autrement dit, une balance entre compétence et défi nécessaire à l'exercice du jeu.

Lorsque ces deux barrières de l'investissement et de l'accès tendent à disparaitre, alors l'utilisateur commence à se sentir engagé. Un joueur engagé est intéressé par le jeu et souhaite continuer à jouer. Ce qu'il manque néanmoins à cette expérience, c'est ce degré d'attachement émotionnel que l'on retrouve dans les derniers niveaux de l'immersion. (Brown et Cairns, 2004, p. 1299, notre traduction)

Le deuxième niveau, l'absorption (engrossment) sous-entend la construction du jeu et un investissement émotionnel de la part du joueur : « c'est lorsque les caractéristiques du jeu se combinent afin que les émotions du joueur soient directement affectées par le jeu » (Brown et Cairns, 2004, p. 1299, notre traduction). Le troisième niveau, ou « immersion totale ", équivaut à un sentiment de présence dans le jeu, sentiment lié à l'atmosphère et l'empathie ressentie. Les auteurs soulignent que cette immersion n'est pas étrangère au concept de flow, mais nous pourrions ajouter que toute leur approche de l'engagement y fait écho en détaillant différentes étapes de l'expérience où un équilibre entre l'expérience du joueur et le système de jeu est nécessaire pour atteindre l'immersion totale.

\section{La sémiotique de l'engagement : la notion de « schémas »}

L'idée d'un continuum entre différents types d'engagement était déjà présente chez Douglas et Hargadon (2001), qui font la distinction entre engagement, immersion et flow à partir de la notion de "schémas». Entendus comme étant des structures codifiées productrices de sens, les schémas sont utilisés et réutilisés par les auteurs en littérature ou les concepteurs de jeux vidéo pour la réalisation de leurs œuvres. Il y aurait engagement lorsque les schémas à interpréter par les lecteurs ou les joueurs ne sont pas préalablement connus et exigent, pour ainsi dire, des efforts interprétatifs. A contrario, l'immersion et, ultimement, le flow font état d'une expérience où les schémas sont si bien maîtrisés que l'interprète peut être immergé au point d'en oublier le dispositif.

Lorsqu'il ou elle est immergé(e) dans un texte, les perceptions, réactions et interactions de la lectrice ou du lecteur se déroulent toutes dans le cadre du texte, 
qui lui-même suggère généralement un schéma unique et quelques scripts précis qui vont orienter les interactions. Inversement, dans ce que nous pourrions appeler l'« expérience affective engagée ", des schémas contradictoires ou des éléments qui défient les schémas conventionnels tendent à perturber l'immersion des lecteurs ou lectrices dans le texte, les obligeant à adopter une perspective extra-textuelle sur le texte, ainsi que sur les schémas qui l'ont façonné et les scripts qu'il opère. (Douglas et Hargadon, 2001, p. 155, notre traduction) voire d'immersion, au sein du dispositif technique et sémiotique, il faut jouer sur des leviers extrinsèques tout en rendant les interactions les plus transparentes possible (i.e. limitant le travail interprétatif). Cela se traduit par le recours aux habitudes des joueurs à répondre à certains incitatifs à l'action et la volonté de reproduire des expériences vécues comme ludiques.

\section{Les leviers de l'engagement : de l'habitus ludique à l'expérience fun}

Le fait que la ludification soit de plus en plus présente dans nos sociétés occidentales (et même que le processus de ludicisation y soit de plus en plus important) participe de cette habituation à y répondre et à s'engager dans une action présentant des caractéristiques ludiques. Plus précisément, à force de répéter certaines stratégies ludiques, elles deviennent aisées à comprendre et les actions exigées de la part du joueur-destinataire apparaissent évidentes. Plus ils sont utilisés, plus ces schémas de ludification sont reconnus par les usagers, éduqués (ou conditionnés) à performer la réponse adéquate. Dans la perspective des tenants de la ludification dans le domaine du marketing (Zichermann, Jo Kim, Werbach, Chou, Burke, etc.), les stratégies ludiques sont alors vues comme un des moyens extrinsèques permettant d'induire une forme d'engagement dans 
une activité voulue. On peut parler de ludification béhaviorale pour définir cette manière de considérer la ludification comme forme possible de conditionnement des comportements.

Or, ce qui semble être une «recette magique» pour s'assurer de l'engagement du consommateur ou du travailleur a été largement critiqué, d'abord pour sa vision trop réductrice de la complexité humaine (Bogost, 2011b ; Deterding, 2014 ; Fuchs et al., 2014 ; Robertson, 2010), mais également parce que les effets de la ludification béhaviorale ne durent qu'à court terme (Rigby, 2014). D'ailleurs, constatant que les effets de la ludification béhaviorale n'étaient efficaces que pour un temps limité, les mêmes spécialistes et entreprises de ludification béhaviorale ont fait évoluer leurs discours quelques années seulement après avoir annoncé la «révolution» de la ludification (Zichermann et Linder, 2013).

En effet, une nouvelle manière de considérer la ludification présente la motivation et l'engagement à partir d'une promesse moins extrinsèque de plaisir. "Il semble que le moteur le plus puissant de l'engagement des joueurs ne soit pas basé sur des méthodes de quantification artificiellement imposées dans tous les contextes possibles, mais bien sur le "fun" » (Fizek, 2014, p. 275, notre traduction). Ce fun ou plaisir est alors défini comme une réaction émotionnelle agréable produite par la capacité d'être engagé dans un comportement ludique qui émerge d'une interaction avec le jeu ou les stratégies de ludification (Fizek, 2014, p. 279). On parle alors de fun-driven mechanisms pour créer une expérience de jeu signifiante suffisamment plaisante et agréable pour susciter l'action (Salen et Zimmerman, 2003, p. 165). D'autres concepts tels que le playfulness (Fizek, 2014) ou le gamefulness (Deterding et al., 2011; Deterding et al., 2014) sont utilisés pour caractériser cette expérience plaisante, résultant d'une manière de concevoir qui tient compte de l'espace de liberté accordé au joueur et qui favoriserait son engagement de manière plus pérenne.

En considérant le glissement possible d'une motivation extrinsèque vers une motivation intrinsèque de plaisir et de satisfaction personnelle, on peut qualifier le type d'engagement de l'individu. Celui-ci passerait en effet d'un engagement béhavioral à un engagement "optimal ", où l'investissement dans un comportement se combine avec un investissement plus affectif. Toutefois, si la ludification peut susciter l'engagement dans une activité à force de répétition des mêmes marqueurs, mécanismes et stratégies ludiques, encore faut-il que l'utilisateur-joueur se laisse convaincre de l'intérêt de performer l'action. La ludification se distingue alors en ce qu'elle est à la fois le contenu du discours, en tant qu'argument pour convaincre de faire une action (" ceci est un jeu»), et la forme même du discours, comme organisation langagière propre au ludique (recours à des schèmes ludiques).

\section{Rhétorique procédurale de l'engagement ludique}

Si, dans les sections précédentes, la ludification a été présentée comme un argument persuasif afin de stimuler l'engagement, de la promesse d'une récompense (motivation extrinsèque) à une expérience plaisante ou satisfaisante (motivation intrinsèque ou flow), il convient maintenant de la définir également comme une forme discursive, c'est-à-dire un ensemble de signes dont le sens réfère à l'univers du jeu. Ces signes, organisés en tant que marqueurs, mécaniques ou stratégies ludiques, s'inscrivent dans des discours plus généraux de représentation du monde et visent à persuader l'individu de performer 
certaines actions (de « jouer le jeu»). Cependant, ils peuvent aussi avoir comme dessein de convaincre de la pertinence, justesse et véracité de messages sous-jacents. Cet art de la persuasion, ou rhétorique, devient particulièrement efficace dans le domaine ludique, puisque le jeu, par définition basé sur la performance de certaines actions, amène l'individu à agir en ne prenant pas nécessairement pleine conscience des discours véhiculés. En définissant la manière dont l'individu doit se conduire pour aboutir à un certain résultat, ce type de rhétorique, appelée "procédurale», peut le convaincre du bien-fondé des actions en lui faisant justement performer ces actions.

En études du jeu, Ian Bogost $(2008,2011$ a) a été le premier à faire valoir le pouvoir de la rhétorique procédurale des jeux vidéo. Cette rhétorique implique de poser un nombre donné d'actions dans un certain ordre afin d'accomplir certains objectifs. Plus précisément, des opérations logiques organisent le sens des actions performées par les joueurs en visant un but à atteindre, que ce but soit celui propre au jeu ou que d'autres buts aient animé les concepteurs. En ce sens, la rhétorique procédurale vise à convaincre d'agir au travers de représentations et d'interactions reposant sur un système de règles, plutôt qu'au travers de mots, d'écrits, d'images ou d'images animées (Egenfeldt-Nielsen, Smith, et Tosca, 2015, p. 161).

En se basant sur le "faire faire », la ludification a recourt à une rhétorique procédurale afin de convaincre de s'engager dans certaines activités, les leviers ludiques se situant dès lors sur la gradation médiane de la motivation (cf. supra). Dans ce cas, tant que la forme sémiotique demeure apparente, c'est-à-dire tant que les marqueurs, mécaniques et stratégies ludiques utilisés afin de motiver l'engagement sont reconnus comme tels, l'individu peut avoir une distance critique par rapport à son activité, distance nécessaire à l'exercice même du jeu (Henriot, 1989, p. 149). Il n'y a alors pas nécessairement de consentement de l'individu à un système de valeurs auquel la ludification donnerait forme, mais plutôt une satisfaction personnelle à s'engager afin d'atteindre les objectifs ludiques du système.

Afin d'illustrer ce point, nous pouvons prendre comme premier exemple celui de Foldit, qui signifie littéralement " pliez-la ». Foldit est un dispositif ludique créé à l'Université de Washington. Le département de biochimie faisait face à un " casse-tête » médical vis-à-vis d'une protéine jouant un rôle important dans la reproduction du VIH. Les chercheurs avaient besoin de pouvoir littéralement "déplier» cette protéine afin de trouver un traitement adéquat pour lutter contre son action. Pour résoudre ce problème, ils ont décidé, avec l'aide du Center for Game Science du département d'informatique, de transformer leur outil de visualisation 3D en un "puzzle compétitif » accessible en ligne. La version bêta du jeu fût lancée en mai 2008 et, quelques semaines plus tard, l'enzyme a été complètement mise à plat par les équipes de joueurs qui s'étaient inscrits sur la plateforme. L'expérience, qui a d'ailleurs donné lieu à deux publications majeures ${ }^{7}$, démontre qu'il est possible d'utiliser l'habitus ludique (cf. supra) de certains joueurs pour résoudre des problèmes concrets. Ce cas peut être considéré comme un exemple de ludification procédurale alors que l'argument pour susciter l'engagement est «le plaisir de résoudre un puzzle ", sans que l'adhésion à un autre argument, extérieur au contexte du jeu, soit nécessaire (e.g. "participer au développement de la science médicale »). L'important était de réussir à "faire faire " un ensemble d'opérations complexes à un grand nombre de personnes, sans qu'elles ne doivent connaitre pour autant les principes médicaux sous-jacents, ni nécessairement vouloir favoriser un mieux-être collectif. 
Un autre exemple vient de l'EcoChallenge organisé depuis plusieurs années par le Northwest Earth Institute ${ }^{8}$. La plateforme propose de créer ${ }^{9}$ ou de rejoindre un événement existant dont l'objectif est, durant une période donnée (minimum quinze jours), de se fixer des " challenges » journaliers visant à réduire sa consommation d'eau, ses émissions de $\mathrm{CO}_{2}$, sa production de déchets, etc., de manière collaborative (en équipes). Telle une compétition sportive, les équipes participantes remportent des points et montent dans des classements, tout en contribuant à une mesure d'impact collective. Chaque participant est régulièrement invité sur la plateforme à partager (textuellement ou en vidéo) son expérience et ses exploits afin d'inspirer les autres participants (ce que le « jeu » reconnaît également sous forme de points/badges gagnés). La rhétorique n'est pas ici fondée sur des arguments scientifiques écologiques, mais bien sur le caractère engageant de la démarche en récompensant directement chaque action. Le système BLAP (Badges, Levels/leaderboards, Achievement, Points) décrit par Nicholson (2012) résume bien la simplicité de ces mécaniques ludiques procédurales. Or, sans internalisation du discours écologique, les défis relevés ne risquent d'animer les participants que de manière brève puisque, comme nous l'avons mentionné, les effets de la motivation extrinsèque illustrée par le système BLAP disparaissent à court terme.

\section{Rhétorique processuelle de l'engagement ludique}

31 Afin de s'assurer des effets à long terme de la ludification, des ressorts propres à la motivation intrinsèque ou, du moins, à une motivation de type régulation identifiée (Deci et Ryan, 2000) doivent être activés et trouver écho chez les participants-joueurs. Que ce soit par répétition d'un argument qui finit par être assimilé plus ou moins consciemment ou parce que cet argument fait écho à un système de valeurs auquel adhère déjà l'individu, les messages véhiculés par la ludification peuvent finir par être internalisés par l'usager. Cette internalisation réfère en fait à l'« adhésion » à un discours - dans le sens de "se déclarer d'accord avec une idée » - terme ici favorisé pour faire état d'un stade où l'individu est non seulement investi d'un point de vue comportemental (en exécutant une série d'actions), mais également d'un point de vue affectif. L'utilisation de ce mot a le double avantage de le distinguer de l'immersion, l'incorporation, l'absorption ou la présence, ainsi que d'insister sur la perspective sémiotique adoptée dans cet article.

Ce processus d'adhésion d'un schème discursif à son univers référentiel peut être encouragé par ce que nous appelons la « rhétorique processuelle». Si la première forme de rhétorique (i.e. procédurale) agit sur les comportements, la seconde agit sur l'identité du récepteur en influençant ou confortant ses valeurs. En usant de stratégies de persuasion qui agissent sur l'individualité, il est alors possible d'influencer l'individu en le "faisant être ", jusqu'à l'adhésion totale où il n'est plus possible de distinguer ce qui relève de l'individu de ce qui provient d'une autorité extérieure. En d'autres termes :

La rhétorique procédurale "fait faire» des actions au joueur (des actions qu'il performe dans la mesure où il est convaincu de l'atteinte d'un objectif) tandis que la rhétorique processuelle «fait être » le joueur (elle lui intime de devenir «quelque chose » en exigeant une adhésion à des idées ou valeurs plus profondes) (Bonenfant et Arsenault, 2016).

Dans le cas de la rhétorique processuelle, la forme sémiotique ludique devient invisible pour l'interprète: les marqueurs, mécaniques et stratégies ludiques ne sont plus reconnus comme tels puisque ce n'est plus le sens de la ludification qui prédomine, mais 
les messages véhiculés. Il ne s'agit plus de " jouer le jeu », mais, par persuasion, d'adhérer à un discours sous-jacent. Le pouvoir de conviction de la ludification paraît ainsi dans toute sa puissance: à la fois comme source de motivation intrinsèque liée à une satisfaction personnelle ou à une adhésion à un discours, à la fois comme forme de rhétorique faisant mouvoir les individus, jusqu'à la possibilité d'agir sur leur individualité par une internalisation des discours véhiculés.

Un exemple emblématique de cette dynamique est la carte de fidélité. Pouvant être considérée comme le « degré zéro » des stratégies de ludification (accumuler des points et recevoir une récompense), elle s'est tellement répandue dans le domaine du marketing que les usagers ne reconnaissent plus cette mécanique comme étant propre au jeu. S'ils pouvaient à l'origine se questionner sur le sens du dispositif, ils sont désormais conditionnés à sortir leur carte, enregistrer les points et réclamer leurs récompenses. Cet habitus, d'abord stimulé par motivation extrinsèque, a fini par convaincre par rhétorique procédurale d'adopter certains comportements. Or, ceux-ci perdurent à partir du moment où l'individu est persuadé du bien-fondé du système dans lequel les cartes s'inscrivent. Le message véhiculé par cette stratégie de ludification, c'est-à-dire que plus grande sera la consommation, plus grands seront les "bénéfices", vise l'adhésion du consommateur au discours capitaliste de manière plus ou moins consciente, jusqu'à en oublier les conséquences d'une surconsommation et accepter de céder une part de sa vie privée pour recevoir de banales récompenses (Coll, 2014).

Si les cartes de fidélité peuvent avoir des effets sur les comportements, voire favoriser une adhésion à un système de valeurs capitalistes, il est possible de considérer ce conditionnement dans d'autres contextes. Par exemple, ce même type de conditionnement peut viser l'acquisition de saines habitudes de vie ou un mieux-être individuel. Le cas de l'application mobile PainSquad développée pour l'hôpital pour enfants malades de Toronto ${ }^{10}$ est particulièrement révélateur à cet égard. Des enfants, sous traitement pour lutter contre leur cancer, doivent tenir le rôle d'enquêteur afin d'investiguer leur propre douleur. L'équipe médicale cherchait en effet un moyen pour motiver ces enfants affaiblis à documenter fréquemment le siège et l'intensité de leur douleur, afin d'adapter leurs traitements. Outre le déclenchement régulier d'enquêtes sur l'application mobile, conduisant à la montée en grade de l'enfant-enquêteur au fur et à mesure de ses investigations, les développeurs ont fait appel à des actrices et acteurs de séries policières populaires au Canada pour enregistrer des mini-séquences vidéo d'encouragements, ce qui contribue à la plongée fictionnelle des enfants dans l'univers des séries policières qu'ils connaissent. Sans les récompenses générées par les mécanismes ludiques, il est très incertain que les enfants aient eu la motivation de documenter leur douleur régulièrement et de manière durable. Or, en motivant le geste, ce comportement peut non seulement devenir routinier, mais les enfants peuvent également en apprécier les bénéfices à long terme grâce à un traitement plus adapté. En ce sens, Painsquad peut être compris comme une forme de rhétorique d'abord procédurale agissant par motivation extrinsèque jusqu'à une internalisation de la motivation prenant la forme d'une rhétorique processuelle agissant sur l'individualité des enfants, désormais convaincus de la nécessité de documenter leur douleur pour viser leur mieux-être, jusqu'à ce que la caractéristique ludique de l'application devienne facultative. 


\section{Conclusion : vers une éthique de la rhétorique ludique} parallèle les mêmes leviers de l'engagement où, par motivation d'abord extrinsèque, est adopté un comportement jusqu'à l'internalisation d'un discours qui peut finalement se suffire à lui-même pour stimuler l'activité. Toutefois, si, d'un côté, il s'agit d'augmenter la consommation et, de l'autre, de favoriser le bien-être des enfants, les messages véhiculés dans ces deux exemples n'ont pas les mêmes valeurs, dans le sens éthique du terme. Ce qui est promu sous le couvert de la ludification est à juger à l'aune de ses effets sur les individus, la société et l'environnement. Ainsi, une réflexion sur l'utilisation de la ludification doit être soulevée de manière plus problématique, en appelant à une éthique de la rhétorique ludique pour favoriser l'engagement, surtout lorsque la question de l'adhésion à un discours est posée. il serait fallacieux, pour reprendre les termes de Mauco (2012), de croire qu'en réponse chaque utilisateur vive une expérience à la fois ludique et plaisante. La phénoménalité du jeu (Henriot, 1989, p. 125), son apparition à la conscience, est évidemment largement arbitraire (p.219). Cependant, il est tout autant questionnable de croire que le recours à des schémas ludiques connus n'agisse pas sur les mécanismes d'action procéduraux et, à terme, ne puisse influer sur l'adhésion d'un individu aux valeurs et discours véhiculés par un dispositif ludifié. Tel que souligné précédemment, tant que les marqueurs, mécaniques et stratégies ludiques demeurent apparents, l'individu peut conserver cette nécessaire distance par rapport à la pratique ludique (p. 149). Or, le risque est de négliger la pratique éthique, l'autonomie et l'auto-réflexion, en cherchant à exploiter les mécanismes ludiques sans prise de conscience «pour les mettre en œuvre, pour les exécuter en tant que répétition mécanique de tâches » (Sicart, 2014, p. 234, notre traduction). La nécessité de maintenir une distance critique propre à l'exercice du jeu est certainement un point d'achoppement de toute réflexion sur la ludification, qui n'est justement pas un jeu en ce qu'elle cherche généralement à devenir un outil de rhétorique.

L'exemple du simulacre de « jeu » Beat up Anita Sarkeesian (2012) illustre bien la réflexion éthique nécessaire à toute manifestation de ludification. Celui-ci a été hébergé (il ne l'est plus) sur la plateforme newsgrounds.com quelque temps avant ce qui a été appelé le « gamergate $»^{11}$. Le principe était assez rudimentaire, puisqu'il fallait « simplement » cliquer sur le portrait de la blogueuse féministe. Tout en entendant certaines de ses répliques sur l'immaturité des gamers les plus décriées par certains joueurs, son visage paraissait progressivement se tuméfier comme si elle avait été battue physiquement. Sans entrer dans le débat sur le fond de l'affaire, il est clair que, si cette simple mécanique procédurale peut repousser l'individu choqué par cette violence, elle peut également avoir pour effet d'induire à terme une adhésion processuelle au discours: ce type de dispositif pourrait permettre de renforcer chez certains la légitimité de la violence naissante à l'encontre de la bloggeuse, voire de la violence faite aux femmes en général.

D'autres exemples pourraient être développés afin de souligner cette zone floue existant entre une motivation intrinsèque, un engagement total dans l'activité ludifiée et l'adhésion à un système de valeurs par internalisation d'un discours. Au travers de notre cadrage conceptuel et des quelques exemples traités, notre intention, dans cet article, était avant tout de montrer l'importance de problématiser la question de l'engagement et 
de l'adhésion au travers de mécanismes de ludification. L'objectif n'était donc pas de démontrer que la ludification fonctionne ou ne fonctionne pas, ou même qu'il est " bien " ou «mal » d'y avoir recours, mais de questionner à la fois la ludification en tant que discours et l'usage qui en est fait. Dans la perspective actuelle de la ludification, le jeu n'est pas vu pour son caractère autotélique, mais prend la forme d'une rhétorique visant à convaincre de la légitimité de valeurs et de comportements qui les performent. $\mathrm{Si}$, comme le défend Henriot $(1969,1989)$, le jeu est de l'ordre de l'idée, ce glissement rhétorique est loin d'être anodin, d'autant plus dans une société de plus en plus ludicisée (Raessens, 2014). L'engagement par la ludification devient dès lors un véritable enjeu éthique.

\section{BIBLIOGRAPHIE}

BERGER M. et DE MUNCK J. (2015), « Présentation. Participer, entre idéal et illusion » Recherches sociologiques et anthropologiques, vol. 46, $\mathrm{n}^{\circ} 1, \mathrm{pp} .1-24$.

BOGOST I. (2008), « The Rhetoric of Video Games », in K. Salen (ed.), The Ecology of Games: Connecting Youth, Games, and Learning, Cambridge, The MIT Press, pp. 117-140.

BOGOST I. (2011a), « Persuasive Games: Exploitationware », Gamasutra http:// www.gamasutra.com/view/feature/134735/persuasive_games_exploitationware.php?page=2

BOGOST I. (2011b), « Gamification Is Bullshit », The Atlantic. https://www.theatlantic.com/ technology/archive/2011/08/gamification-is-bullshit/243338/

BONENFANT M. et ARSENAULT D. (2016), « Dire, faire et être par les jeux vidéo », Implications philosophiques, http://www.implications-philosophiques.org/ethique-et-politique/ethique/direfaire-etre-ethique-performativite-et-rhetoriques-procedurale-et-processuelle-dans-les-jeuxvideo/

BONENFANT M. et GENVO S. (2014), « Une approche située et critique du concept de gamification », Sciences du jeu, n², https://journals.openedition.org/sdj/286

BRAULT-LABBÉ A. et DUBÉ L. (2012), « Mieux comprendre l'engagement psychologique : revue théorique et proposition d'un modèle intégratif », Les Cahiers Internationaux de Psychologie Sociale, $\mathrm{n}^{\circ} 81, \mathrm{pp} .115-131$.

BROUGÈRE G. (2005), Jouer/Apprendre. Paris, Economica.

BROWN E. et CAIRNS P. (2004), «A Grounded Investigation of Game Immersion », dans CHI '04 Extended Abstracts on Human Factors in Computing Systems. New York, ACM, pp. 1297-13.

BUCY E. P. et GREGSON K. S. (2001), « Media Participation: A Legitimizing Mechanism of Mass Democracy », New Media \& Society, vol. 3, n³, pp. 357-380.

BURKE B. (2014), Gamify: How Gamification Motivates People to Do Extraordinary Things, Brookline, Routledge.

CALLEJA G. (2011), In-Game: From Immersion to Incorporation. Cambridge (MA), The MIT Press. 
CHABOT P.-L. (2012), De l'immersion à l'engagement, la perspective des concepteurs de jeux vidéo sur l'expérience de jeu. Mémoire présenté à la Faculté des arts et des sciences en vue de l'obtention du grade de M. Sc. en sciences de la communication, Montréal, Université de Montréal.

CHATFIELD T. (2010), Fun Inc.: Why Gaming Will Dominate the Twenty-First Century, New York, Pegasus Books.

CHOMSKY N. (1969), Aspects of the Theory of Syntax, Cambridge (MA), The MIT Press,

COLL S. (2014), Surveiller et récompenser. Les cartes de fidélité qui nous gouvernent, Genève, Éditions Seismo.

COWLEY B., CHARLES D., BLACK M. et HICKEY R. (2008), « Toward an Understanding of Flow in Video Games », Video Games \& Computer Entertainment, vol. 6, n² 2, 20:1-20:27.

CSIKSZENTMIHALYI M. (1997), Finding Flow: The Psychology of Engagement with Everyday Life, New York, Basic Books.

CSIKSZENTMIHALYI M. (2008), Flow: The Psychology of Optimal Experience, New York, Harper Perennial Modern Classics.

DETERDING S. (2014), « Eudaimonic Design, or: Six Invitations to Rethink Gamification ». Dans Fuchs M, Fizek S, Ruffino P et Schrape N (Éd.), Rethinking Gamification, Lüneburg, Meson-Press, pp. 305-331.

DETERDING S., DIXON D., KHALED R. et NACKE L. (2011), « From game design elements to gamefulness: defining "gamification" ". In Proceedings of the 15th International Academic MindTrek Conference : Envisioning Future Media Environments, New York, ACM, pp. 9-15.

DETERDING S., DIXON D., KHALED R. et NACKE L. (2014), « Du game design au gamefulness : définir la gamification », Sciences $d u$ jeu, $n^{\circ}$ 2, https://journals.openedition.org/sdj/287

DOUGLAS Y. et HARGADON A. (2000), « The Pleasure Principle : Immersion, Engagement, Flow », in Proceedings of the Eleventh ACM on Hypertext and Hypermedia. New York, ACM, pp. 153-160.

DOUGLAS Y. et HARGADON A. (2001), « The pleasures of immersion and engagement: schemas, scripts and the fifth business ", Digital Creativity, vol 12, n 3, pp. 153-166.

EGENFELDT-NIELSEN S., SMITH J. H. et TOSCA S. P. (2015), Understanding Video Games: The Essential Introduction, Londres, Routledge.

ERMI L. et MÄYRÄ F. (2005), « Fundamental Components of the Gameplay Experience: Analysing Immersion ", In Proceedings of the 2005 DiGRA International Conference: Changing Views: Worlds in Play, http://citeseerx.ist.psu.edu/viewdoc/download?doi=10.1.1.103.6702\&rep=rep1\&type=pdf

FIZEK S. (2014), « Why fun matters : in search of emergent playful experiences », in Fuchs M, Fizek S, Ruffino P, et Schrape N (eds), Rethinking Gamification, Lüneburg, Meson-Press, pp. 273-287. FUCHS M., FIZEK S., RUFFINO P. et SCHRAPE N. (eds) (2014), Rethinking gamification. Lüneburg, Meson-Press.

GENVO S. (2012), « La théorie de la ludicisation : une approche antiessentialiste des phénomènes ludiques ", communication présentée à Journée d'études Jeu et jouabilité à l'ère numérique, http:// www.ludologique.com

GENVO S. (2013), « Penser les phénomènes de ludicisation à partir de Jacques Henriot », Sciences du jeu, $\mathrm{n}^{\circ} 1$, https://journals.openedition.org/sdj/251

HENRIOT J. (1969), Le Jeu, Paris, PUF.

HENRIOT J. (1989), Sous couleur de jouer, Paris, Jose Corti. 
HUIZINGA J. (2008 [1938]), Homo ludens : essai sur la fonction sociale du jeu, Paris, Gallimard.

KOSTER R. (2005), A theory of fun for game design, Scottsdale, Paraglyph Press.

LEIBNIZ W. G. (1993), Discours de métaphysique [1686], Paris, Presses pocket.

MAUCO O. (2012), Sur la Gamification, http://www.gameinsociety.com/post/2012/01/19/Sur-lagamification2

McGONIGAL J. (2011), Reality is Broken: Why Games Make Us Better and How They Can Change the World, Londres, Vintage Books.

McMAHAN A. (2003), « Immersion, Engagement, and Presence: A method for analyzing 3-D Video Games » in M. J.P. Wolf et B. Perron (eds), The Video Game Theory Reader, Londres, Routledge, pp. 67-86.

NICHOLSON S. (2012), « Strategies for meaningful gamification: Concepts behind transformative play and participatory museums ", communication à Meaningful Play 2012, Lansing, Michigan, http://scottnicholson.com/pubs/meaningfulstrategies.pdf

PHILIPPETTE T., COLLARD A.-S. et KLEIN A. (2016), « L'économie collaborative : entre jeu, participation et confiance », Recherches en Communication, vol. 42, pp. 189-202.

POELS K., DE KORT Y. et IJSSELSTEILN W. (2007), « It is Always a Lot of Fun! Exploring Dimensions of Digital Game Experience Using Focus Group Methodology », in Proceedings of the 2007 Conference on Future Play. New York, ACM, pp. 83-89.

RAESSENS J. (2014). The Ludification of Culture. Dans Fuchs M., Fizek S., Ruffino P., et Schrape N. (Éd.), Rethinking Gamification, Lüneburg, Meson-Press, pp. 91-114.

RIGBY S. C. (2014), « Gamification and motivation », in The Gameful World, Cambridge, MIT Press, pp. 113-137.

ROBERTSON M. (2010), « Can't play, won't play », Hide\&Seek, http:// www.hideandseek.net/2010/10/06/cant-play-wont-play/

RYAN R. M. et DECI E. L. (2000), « Self-determination theory and the facilitation of intrinsic motivation, social development, and well-being », The American Psychologist, vol. 55, n 1, pp. 6878.

SALEN K. et ZIMMERMAN E. (2003), Rules of Play: Game Design Fundamentals, Cambridge (MA), MIT University Press.

SHERNOFF D., CSIKSZENTMIHALYI M., SHNEIDER B., et SHERNOFF E. (2003), « Student Engagement in High School Classrooms from the Perspective of Flow Theory », School Psychology Quarterly, vol. $18 \mathrm{n}^{\circ}$ 2, pp. 158-176. https://doi.org/10.1521/scpq.18.2.158.21860

SICART M. (2014), «Playing good life : gamification and ethics », The Gameful World, Cambridge, MIT Press, pp. 225-244.

SILVA H. (2013), « La "gamification" de la vie : sous couleur de jouer ? », Sciences du jeu, n 1, https://journals.openedition.org/sdj/261

SKALSLI P., LANGE R. et TAMBORINI R. (2007), « Mapping the way to fun: The effect of video game interfaces on presence and enjoyment ", communication à the International Communication Association Conference, San Francisco, CA, May 2007 http://citeseerx.ist.psu.edu/viewdoc/ download?doi=10.1.1.524.3016\&rep=rep1\&type=pdf 
SLATER M., LINAKIS V., USOH M., KOOPER R. et STREET G. (1996), « Immersion, Presence, and Performance in Virtual Environments : An Experiment with Tri-Dimensional Chess ", ACM Virtual Reality Software and Technology, pp. 163-172.

THERRIEN C. (2011), Illusion, idéalisation, gratification. L'immersion dans les univers de fiction à l'ère du jeu vidéo, Thèse de doctorat en sémiologie, Université du Québec à Montréal.

WERBACH K. et HUNTER D. (2012), For the Win. How Game Thinking Can Revolutionize Your Business, New York, Wharton Digital Press.

ZICHERMANN G. et CUNNINGHAM C. (2011), Gamification by Design: Implementing Game Mechanics in Web and Mobile Apps, Sebastopol, O’Reilly Media.

ZICHERMANN G. et LINDER J. (2013), The gamification revolution: how leaders leverage game mechanics to crush the competition, New York, McGraw-Hill Education.

\section{NOTES}

1. La notion de dispositif pourrait être longuement discutée. Elle nous permet ici de prendre en compte des cas qui sont soit des objets médiatiques particuliers (e.g. site web, communauté en ligne et application mobile), soit des situations de médiation pouvant inclure différents objets médiatiques (e.g. exposition et classe).

2. "The leader in entreprise gamification », voir https://badgeville.com, consulté le 21 novembre 2017.

3. «motivate[s] and engage[s] your team with gamification", voir https://www.hoopla.net, consulté le 21 novembre 2017.

4. Gabe Zichermann est une des figures de proue du mouvement de la gamification aux ÉtatsUnis. Il est notamment à l'initiative de la conférence annuelle Gamification Summit (GSummit).

5. "We handcraft gamified experiences around your brand's dna», voir http://dopa.mn, consulté dernièrement le 21 novembre 2017.

6. À noter que Calleja (2011) préfère également le terme anglais involvement pour faire état de l'engagement du joueur, mais introduit quant à lui le concept d'incorporation au lieu de celui, plus polysémique, d'immersion. Pour plus de détails, voir notamment l'article d'olivier Caïra dans ce même numéro.

7. Voir https://www.scientificamerican.com/article/foldit-gamers-solve-riddle/ et http:// www.pnas.org/content/108/47/18949.full, consultés le 25 novembre 2017.

8. Voir https://ecochallenge.org/, consulté le 25 novembre 2017

9. Service payant.

10. http://www.sickkids.ca/Research/I-OUCH/Pain-Squad-App/index.html, consulté le 26 novembre 2017.

11. Pour plus d'informations à ce sujet, voir notamment http://www.lemonde.fr/pixels/ article/2014/09/15/derriere-le-gamersgate-un-groupe-antifeministe_4485191_4408996.html, consulté le 27 novembre 2017. 


\section{RÉSUMÉS}

Cet article questionne les liens entre engagement et types de motivation, en problématisant la manière dont la ludification cherche à stimuler la participation et la poursuite d'une activité dont les objectifs sont étrangers au jeu. Sur un plan théorique, il s'agit de lier le concept de ludification avec la notion d'engagement afin d'évaluer la manière dont les marqueurs, mécaniques et stratégies ludiques peuvent agir comme motivateurs à l'action, voire susciter l'adhésion à certaines idées. Sur un plan plus pratique, il s'agit de relever les éléments sémiotiques et discursifs de ces dispositifs ludifiés qui agissent comme des formes de rhétorique procédurale (faire faire) ou processuelle (faire être). La ludification a en effet la spécificité de jouer sur un double registre: elle est à la fois le contenu du discours, en tant qu'argument pour convaincre de poser une action, et la forme même du discours, comme organisation sémiotique de ces actions.

This article questions the links between engagement and different kinds of motivation to act, by problematizing the way in which gamification or ludification seeks to stimulate participation and the pursuit of an activity that is not a game. From a theoretical viewpoint, we consider the concept of gamification in the light of the notion of engagement to evaluate the way in which game markers, mechanics and strategies can play the role of motivators for action, and even encourage adherence to ideas. From a more practical viewpoint, we try to identify semiotic and discursive elements of gamified systems that work as procedural (doing) or processual (being) rhetoric. Indeed, gamification has the specificity of playing a double-dealing: it is at the same time the content of the discourse, as an argument to convince to act, and the very form of it, as a semiotic organization of those actions.

\section{INDEX}

Mots-clés : engagement, ludification, jeu, rhétorique, adhésion, éthique

Keywords : engagement, gamification, game, rhetoric, adherence, ethics

\section{AUTEURS}

\section{MAUDE BONENFANT}

Université du Québec à Montréal

THIBAULT PHILIPPETTE

Université Catholique de Louvain 